\title{
Negotiation 2.0
}

\author{
Elizabeth A Judson \& Johannes Dapprich \\ Business negotiations can be tricky, but with the help of some specialized software the process can be streamlined.
}

\begin{abstract}
If you're an academic researcher who has hit upon a breakthrough in the lab that you intend to commercialize with company funding, or if your company is looking for an academic partner to conduct preclinical work or advance a product into human trials, you'll be working with another institution's technology transfer office to try and make this happen. The problem is that many collaborations are delayed, stifled or even fall apart at the outset due to an inability of the participating entities to resolve terms and conditions in the negotiation of the contract required for sponsored research.

To help with the negotiation process in the United States, there is TurboNegotiator, a tool developed by the University-Industry Demonstration Partnership (UIDP). The program-free to UIDP members-can streamline the interaction between university and industry and can help you quickly identify where you have agreement and what areas still need additional work or compromise. To our knowledge, there is no other program like TurboNegotiator currently available.
\end{abstract}

\section{Talking can be hard to do}

Universities and companies have very different structures and cultures that sometimes may seem almost incompatible. Academic culture typically emphasizes unbounded thinking, the free exchange and dissemination of ideas, and fundamental research. In contrast, industrial R\&D centers around a specific purpose and goal, efficiency and maintaining focus, and profitability. The

Elizabeth A. Judson is program manager of TurboNegotiator at University-Industry Demonstration Partnership, Washington, DC, USA. Johannes Dapprich is research director at Generation Biotech, Lawrenceville, New Jersey, USA.

email: beta@turbonegotiator.orgor

jdapprich@generationbiotech.com

\section{Box 1 Components of a negotiation}

TurboNegotiator helps outline the basics of the negotiating process in the following areas. Background: this section provides information about the research project in general and prior research that may be related.

Nature of project: this section defines the research project as fundamental or applied, defines the expected contributions from each party and outlines how the research outcomes will be used.

Background intellectual property: this section helps identify background intellectual property and confidential information that may be required in the research.

Likelihood of invention: this section frames the potential for various types of intellectual property to be developed during the research and outlines the questions of who will own what and how the end product will be handled.

Publication rights: this section informs the parties about the sponsor's rights to review publication drafts before they are submitted, remove confidential information and identify potentially patentable inventions, and it provides the related periods of time for these processes.

Indemnification: this section sets the expectations of the parties to cover liability resulting from the acts or omissions of their personnel and outlines the related law.

Conflict of interest: this section identifies whether any personnel working on the project may have a conflict of interest.

trick is to get both sides through a negotiation relatively quickly without it blowing up due to some underlying fault that ends up preventing the two sides from connecting in agreement. As in research, half the work toward a solution is defining the problem accurately and fully.

It is absolutely essential at an early stage of negotiations that all individuals within each organization communicate effectively with each other so that internal agreement on open questions is reached quickly before communicating the results to the negotiation partner for resolution ${ }^{1}$.

Even if each institution has the best of intentions, it is possible that somewhere in the internal procedures a disconnect has occurred. If that happens, the proper work flow that is required for a timely resolution breaks down, and, worse, messages sent to the negotiation partner become inconsistent.
Therefore, identifying the right individuals to contact within the other institution early on and understanding their authority and role in decision making is critically important. Any negotiation will run much more smoothly if feedback is obtained directly from decision makers, particularly if it can be done reasonably fast. If no timely and meaningful responses can be obtained from the negotiation partner other than "we are working on it," "we will get back to you" or, worse, "this is not our problem," then this may indicate the inability of the institution to work as a consistent and coherent entity, which is a serious red flag for any continued negotiations.

Another problem that can hinder the ability to form sponsored research agreements is that the time delay before receiving a decision can vary greatly, and negotiations can sometimes approach a full year before any conclusive 


\section{Box 2 Resources for the negotiator}

The 'Knowledge Base' resource on TurboNegotiator can help clarify some terminology.

Contract accords: these are statements of consensus between university and industry on components of a research agreement's terms and conditions.

University-Industry Demonstration Partnership guiding principles: these support productive research collaborations between universities and industry, recognizing that the missions are distinct.

Living case studies: these are examples of research partnership situations and how the guiding principles relate to each case.

Frequently asked questions: these are answers to common questions regarding industrysponsored research at universities.

Bayh-Dole act: this provides information regarding how this law (which covers intellectual property in federally funded research) impacts federal- and industry-sponsored research at universities.

Materials transfer in academia: this is information on why and how the transfer of materials in and out of academia must be documented and handled.

Department of Defense flowdown clauses: these are usually incorporated in industry subcontracts to universities from defense contracts.

approval is granted. Again, a common reason is ineffective, incomplete or nonfunctioning communication among the individuals in one party that is working on a deal.

Try to make sure your team is communicating quickly and clearly, but admittedly this can often seem next to impossible, especially in large organizations. Big institutions typically have a large set of rules in place to ensure the proper execution of all its processes. Those rules and working templates are based on years of experience, are intended to provide both guidance and protection to employees, patients and collaborators, and are meant to facilitate successful research, innovation, publications and patient benefits, etc. Therefore no single person can be expected to hold either the knowledge or authority to constructively work out the framework for a successful collaboration with the other party while accurately conforming to all of the organization's rules.

Impasse situations can arise out of this rather suddenly. For example, the legal department of the firm or university you are dealing with may suddenly find itself in charge of executing the collaboration agreement, but your project may not quite fit within its historical rules. If the person in charge is expected first and foremost to uphold the rules and avoid making any mistake, you will be stuck. Without being able to adjust the rules so that a realistic, reasonable and legal framework can be achieved, negotiations can grind to a halt.

This can be especially painful if such problems are discovered late in the process, when both parties may have already invested considerable resources, time and emotional energy in the collaboration.

At some point, if no real progress can be made, you need to cut your losses and go elsewhere. Companies in particular do not have the benefit of stable grants and tenured positions; instead, they face real deadlines and commitments and therefore cannot afford to wait indefinitely.

Sounds like a lot to handle, doesn't it? The truth is, it can seem nearly impossible to get a deal closed, particularly if you have less negotiating experience than your potential partner. The good news is that there is a software program now available that can provide help.

\section{Finding help}

TurboNegotiator is now available for members of the UIDP free of charge. (Organizations that are not members can request a free trial.) The UIDP has more than 70 members, two-thirds of which are large and small, public and private universities. One-third of the members are companies from biotech, chemical, engineering and information technology industries. The purpose of the UIDP is to increase the value of collaborative partnerships between university and industry in the United States.

Modeled on the 20-year success of the Federal Demonstration Partnership, which streamlined government-university relations, the UIDP advances demonstration projects through working groups committed to principled and transparent negotiations and operations. TurboNegotiator is the UIDP's first demonstration project, and it can help industry and universities manage the negotiation process by providing a checklist of issues necessary to consider and agree upon, both internally and with each other. It also acts as a repository for the parties to maintain a history of their progress; that way if the faces at the negotiating table change, the intellectual capital is not lost. The collaborative software platform was released in pilot form in early 2009.

TurboNegotiator is a software tool that allows the parties in a negotiation to identify areas of commonality and areas that need greater compromise to develop a solution both parties could work with. When the parties focus on the benefits that result from the collaboration and streamline the negotiations, the research can be conducted in a timely manner.

The program provides an overview of all components that may play a role in the negotiation process (Box 1). This allows their quick assessment by each party and thereby informs each side about what terms are negotiable. Speed is essential—quickly identifying the areas that need work significantly increases the comfort level for both parties and assures them that respective goals and expectations eventually will be achieved while at the same time safeguarding fundamental needs such as freedom to publish and protection of underlying IP.

The primary goal of the program is to decrease negotiation time. That said, it can also be an effective tool to train new or lessexperienced negotiators, and it can act as a repository of information to preserve knowledge and creative solutions.

\section{Getting started}

The first step to using the program is to request access. You can do this by e-mailing beta@turbonegotiator.org.

Once accepted, you'll need to use the secure portal for negotiations, which requires a user account and password. (As the security is limited to password-protected accounts, we do not recommend uploading confidential information. Future upgrades will have enhanced security.)

Two user categories permit varying levels of access. UIDP members access TurboNegotiator as 'Negotiation Creators' with the privileges of creating negotiations and inviting participants. 'Negotiation Participants' have access only to the negotiations they have been invited to and are not required to be UIDP members. When a negotiation is created, the participants receive an e-mail invitation and must accept the invitation by clicking on the embedded link to generate the user account and password, which is sent in a separate e-mail message. 


\section{Box 3 Case study: Ivy Leaf Medical and PiccoloBio}

Let's say a small biotech company, PiccoloBio, has been collaborating with academic partners and a large strategic partnering company to commercialize the results of funding from Small Business Innovation Research grants. It has experienced unnecessary costs and delays in the process of setting up a mutually beneficial and sustainable collaboration framework. Stumbling blocks that have to be resolved are the need for protection and control of key trade secret know-how on the company's side along with the goal of having an exclusive right to commercialize any sponsored new intellectual property (IP), versus the freedom to publish, the pursuit of research funding, the gain of downstream royalties and the use of jointly developed technology for in-house purposes on the academic side.

Researchers at a local hospital, Ivy Leaf Medical, are experiencing a common problem, and a significant demand thus exists for a potential solution. PiccoloBio has an approach that can solve the problem but lacks resources to develop and apply the approach for clinical practice. The overall goal is to strike a relationship that protects the company's core IP and its technology's commercial value while allowing the Ivy Leaf researchers to evaluate and use the technology and include it in publications and grant applications. Ideally, the collaboration agreement also should enable a reliable mechanism that allows the parties to jointly advance the technology through fundamental improvements and to develop new applications for it.

Thus a formula has to be found that allows each party to gain in its own preferred way from any benefit of the joint progress that may be achieved, either through work of the company or the institution. These benefits may consist of basic technological improvements, contacts for funding or new applications for the technology, related new IP, potential patent filings or publications, material for marketing or use of jointly created data at conferences for the benefit of the technology as a whole.

When a company is looking to collaborate with a large academic institution, a frequent problem is that the more respected the institution is, the more rigid and immovable it will likely consider its set of rules that govern any interaction with outside parties. The effect is that even though the potential benefits of a strategic collaboration may be recognized and supported at the highest level of the institution, it falls to individuals in the sponsored research or technology transfer offices to work out the details. They may be under immense pressure to get it right and avoid mistakes at all cost in the process, even though a given situation may simply not fit previous experiences - therefore making it essentially impossible to abide exactly by the rules.

As a result, there may not be any response at all from the technology transfer office, even on seemingly simple questions. Even at the director level, the technology transfer office may not be sure who can provide answers or who has the authority to potentially adapt the rules to make them work as intended for a somewhat different situation.

A negotiating tool like TurboNegotiator can help both sides to quickly consider all relevant facets that have to be addressed for an agreement. This avoids critical omissions or misunderstandings that can become more and more difficult to address the longer the negotiation process carries on. If there are additional issues that need to be addressed that are not brought out in the questionnaire, the TurboNegotiator message board can be used to document these areas.
Before logging in to the secure sections of TurboNegotiator, there are many resources available through the 'Knowledge Base' section (Box 2). It includes a glossary outlining how TurboNegotiator defines key concepts throughout the application, the contract accords and other useful information. The 'Welcome Page' provides links to the UIDP, information on obtaining user accounts and contacting the UIDP and a link to a short video demonstration on how to use TurboNegotiator.

Once you've logged in to TurboNegotiator, a list of your negotiations will appear, and you'll have access to the 'Negotiation Portal', an area where you can upload and access files and documents pertinent to the negotiations, post to a message board and fill out the questionnaire. Although TurboNegotiator is currently limited to two parties in a negotiation (the next upgrade to the software will expand to handle more complex negotiations), there is no limit to the number of participants that may be invited to represent each party, so the tool can be used to reach internal consensus.
The software leads you through the sections of the questionnaire, but more experienced negotiators can identify the sections they need to focus on and save time. If necessary, negotiators can upload files for viewing by either party. And although the software was designed under the assumption of US laws and practices, some parts may be useful in negotiations with international organizations.

When comments are posted to message threads or questions, all of the negotiation participants receive e-mail notification. After the questionnaire has been completed, the parties can generate a comparison report that lists all of the questions and answers of both parties.

To illustrate the benefits of using TurboNegotiator, a fictitious case study has been developed illustrating an interaction between a small biotechnology company and a large public research hospital (Box 3).

\section{Conclusions}

TurboNegotiator can provide a wealth of knowledge to people that either do not have experience with negotiating sponsored research agreements or with the commercial restraints and needs of a company. More importantly, TurboNegotiator systematically speeds up the negotiation process by enabling the negotiators to focus on the areas of importance. Although it is not a replacement for direct communication, it will make the communications more efficient — this can be particularly useful by ensuring critically needed consistency within each institution. As long as both parties are willing to use it in their interaction, TurboNegotiator evens the playing field for the parties by providing a neutral platform for negotiations.

1. Slowinski, G. \& Sagal, M. The Strongest Link: Forging a Profitable and Enduring Corporate Alliance (AMACOM, New York, 2003).
To discuss the contents of this article, join the Bioentrepreneur forum on Nature Network: http://network.nature.com/groups/bioentrepreneur/forum/topics 